\title{
Microwave-induced thermoacoustic tomography: Reconstruction by synthetic aperture
}

\author{
Dazi Feng, Yuan Xu, Geng Ku, and Lihong V. Wang ${ }^{\text {a) }}$ \\ Optical Imaging Laboratory, Biomedical Engineering Program, Texas A\&M University, 3120 TAMU, \\ College Station, Texas 77843-3120
}

(Received 14 May 2001; accepted for publication 20 September 2001)

\begin{abstract}
We have applied the synthetic-aperture method to linear-scanning microwave-induced thermoacoustic tomography in biological tissues. A nonfocused ultrasonic transducer was used to receive thermoacoustic signals, to which the delay-and-sum algorithm was applied for image reconstruction. We greatly improved the lateral resolution of images and acquired a clear view of the circular boundaries of buried cylindrical objects, which could not be obtained in conventional linearscanning microwave-induced thermoacoustic tomography based on focused transducers. Two microwave sources, which had frequencies of 9 and $3 \mathrm{GHz}$, respectively, were used in the experiments for comparison. The $3 \mathrm{GHz}$ system had a much larger imaging depth but a lower signal-noise ratio than the $9 \mathrm{GHz}$ system in near-surface imaging. (c) 2001 American Association of Physicists in Medicine. [DOI: 10.1118/1.1418015]
\end{abstract}

Key words: thermoacoustics, tomography, synthetic aperture, microwave

\section{INTRODUCTION}

Microwave-induced thermoacoustic tomography is emerging as a nonionizing imaging modality. When electromagnetic radiation is irradiated upon biological tissues, the resulting heat-related expansion of the tissues produces acoustic waves. From the acoustic signals, we can reconstruct the distribution of electromagnetic absorption in soft tissues. The large differences in electromagnetic absorption in various tissues, which are associated with their physiological and pathological status, provide significant contrasts in imaging. For example, the absorption coefficient in cancerous breast tissue is two to five times greater than that in normal breast tissue, due to the increment of water and sodium bounded within malignant cells. ${ }^{1-3}$ This large difference makes it promising to use microwave-induced thermoacoustic tomography to detect breast cancers.

Microwave-induced thermoacoustic tomography combines the advantages of both pure ultrasound and pure microwave imaging. Traditional imaging technology with pure ultrasound (ultrasonography) offers satisfactory spatial resolution but poor soft-tissue contrast, while pure microwave imaging provides good imaging contrast but barren spatial resolution. ${ }^{4-7}$ Microwave-induced thermoacoustic tomography bridges the gap between them. By integrating ultrasound and microwave technology, microwave-induced thermoacoustic tomography has both satisfactory spatial resolution and good soft-tissue contrast.

In conventional linear-scanning microwave-induced thermoacoustic tomography (LMTT), a focused ultrasonic transducer is used to detect time-resolved acoustic signals. Since the focused transducer has a good response only along the transducer axis, each acoustic signal can be converted into a one-dimensional image. Linear scanning of the ultrasonic transducer yields multiple one-dimensional images, which can be combined to form a two-dimensional image of the sample. ${ }^{8-10}$ In the two-dimensional images obtained with conventional LMTT, only boundaries that are nearly perpendicular to the axis of the ultrasonic transducer can be detected because most of the thermoacoustic waves travel in a small solid angle around the normals of boundaries; spherical or oblique boundaries of buried objects whose thermoacoustic waves have a large angle with the axis of the ultrasonic transducer cannot be imaged because the ultrasonic transducer receives little signal from these boundaries.

To overcome this deficiency of traditional LMTT, we have applied the synthetic-aperture method to LMTT. In this method, thermoacoustic signals were detected from multiple locations and the synthetic delay-and-sum algorithm was then used for the reconstruction of the images. The syntheticaperture method has been applied in PAT previously; ${ }^{11-14}$ and weights were assigned to the signals according to the sensor's directivity to improve the SNR at the expense of lateral resolution. In our experiment, the raw data were obtained by a $2.25 \mathrm{MHz}$ nonfocused transducer instead of a focused one, as in traditional LMTT, and the syntheticaperture reconstruction method based on the delay-and-sum algorithm was applied to reconstruct the images. By applying the synthetic-aperture method, we have improved the lateral resolution of the images and enhanced our ability to image spherical or oblique boundaries in the samples. Images acquired from two microwave sources with different frequencies were compared; the $3 \mathrm{GHz}$ system has a much larger imaging depth but a lower SNR than the $9 \mathrm{GHz}$ system in near-surface imaging.

\section{METHODS}

\section{A. Reconstruction method}

The image reconstruction method is illustrated in Fig. 1. For convenience, we converted both the signal-delay time 


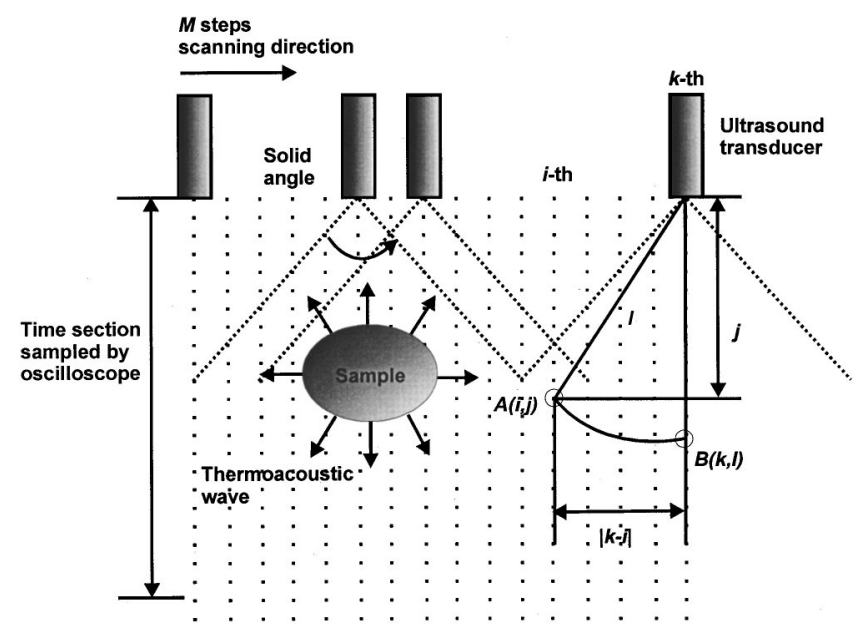

FIG. 1. Scanning and reconstruction method.

and the spatial distance into the number of pixels according to the speed of the acoustic wave traveling in the media. For the $50 \mathrm{MHz}$ sampling rate, $1 \mu \mathrm{s}=50$ pixels $=1.5 \mathrm{~mm}$.

Because the transducer is nonfocused, it receives signals from a larger solid angle than does a focused counterpart. In the reconstruction, we evenly projected the signals to each point within the whole solid angle according to the time delay. The time delay corresponds to the distance from the transducer to the point to which we project the signal. This is the algorithm called "delay and sum." In other words, the signal intensity of each point $A(i, j)$ is the sum of the signals from the transducer at different positions delayed with the transit time from the transducer position to the point. So the signal intensity at any point, $A(i, j)$, can be expressed as

$$
A(i, j)=\sum_{k=0}^{M} B(k, l),
$$

where $B(k, l)$ is the signal intensity coming from the $l$ th pixel point in the signals and from the $k$ th transducer scanning position, $M$ is the total number of steps that the transducer scanned, and $l$ is the distance from the $k$ th transducer scanning position to the point $(i, j)$ :

$$
l=\sqrt{(k-i)^{2}+j^{2}} .
$$

Let us consider a point $\left(i_{1}, j_{1}\right)$ where there is a thermoacoustic source. During the data acquisition, all the detectors will receive signals from this particular point after time delays determined by the above equation. Conversely, in the reconstruction, all the detectors contribute signals to this particular point with the appropriate time delays. On the other hand, for a point $\left(i_{2}, j_{2}\right)$ where there is no source, few detectors contribute signals to this point after time delays determined by the above equation. Consequently, the reconstructed intensity at point $\left(i_{1}, j_{1}\right)$ will be much greater than that at point $\left(i_{2}, j_{2}\right)$. In this reconstruction scheme, the detection can be artificially focused onto any specified point $(i, j)$, which is the basic concept of synthetic aperture.

We attempted to add some corrections to the delay-andsum algorithm but found them unnecessary. For example, we

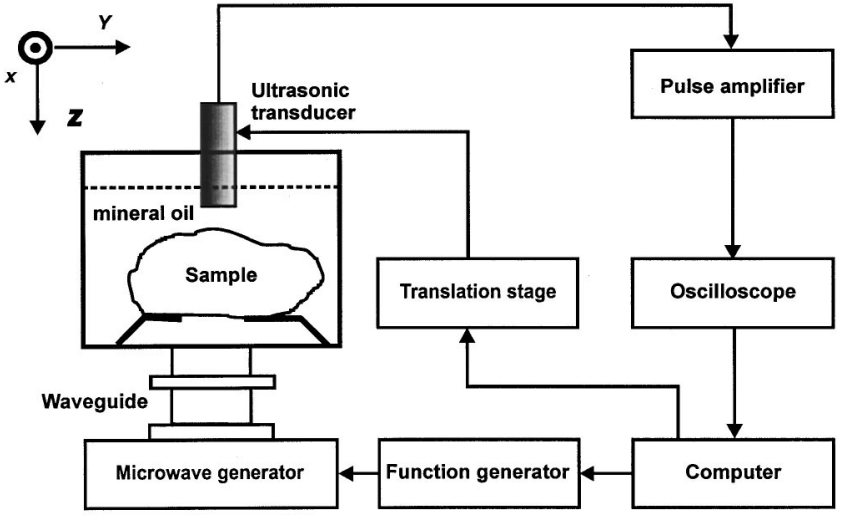

FIG. 2. Experimental setup.

tried applying weights to the signals according to the transducer's directivity, as was done previously. It improved the SNR, but the lateral resolution deteriorated as a result. In our situation, we had already acquired a satisfactory SNR by averaging the thermoacoustic signals 100 to 200 times, indicating that the direct delay-and-sum algorithm works well in LMTT technology.

\section{B. Experimental setup}

The experimental setup for this study is shown in Fig. 2. A Cartesian system was set up for reference. The $z$ axis is along the ultrasonic axis pointing downward. The $x$ axis is perpendicular to the drawing plane and pointed outward. The $y$ axis is in the drawing plane and points to the right.

In the experimental setup, microwave pulses of 9 or 3 $\mathrm{GHz}$, with a width of $0.5 \mu$ s were delivered into the samples. The sizes of the cross section of waveguides were $72 \mathrm{~mm}$ $\times 34 \mathrm{~mm}$ in the $3 \mathrm{GHz}$ system and $23 \mathrm{~mm} \times 10 \mathrm{~mm}$ in the 9 $\mathrm{GHz}$ system. A function generator (DS345, Stanford Research System) was employed to trigger the microwave pulses and synchronize the sampling of an oscilloscope. A linear translation stage (MD2, Arrick Robotics), on which an ultrasonic transducer was mounted, was driven by a computer-controlled stepper motor. The transducer was scanned linearly. The nonfocused ultrasonic transducer (V323, Panametrics) mounted on the translation stage had a central frequency of $2.25 \mathrm{MHz}$ and a $6 \mathrm{~mm}$ diam of an active element. A low-noise pulse preamplifier (500 PR, Panametrics) amplified the acoustic signals coming from the transducer. Then the amplified signals were collected and averaged by an oscilloscope (TDS-640A, Tektronix) and subsequently transferred to a personal computer. The acoustic waves from the sample were coupled to the ultrasonic transducer by mineral oil.

\section{RESULTS AND DISCUSSION}

In this section, we will present and discuss the images acquired from the $3 \mathrm{GHz}$ microwave system and the $9 \mathrm{GHz}$ microwave system, respectively. The first two sets of images were acquired from the $9 \mathrm{GHz}$ system. In the first set of images, we imaged a semicylindrical fat sample with a 


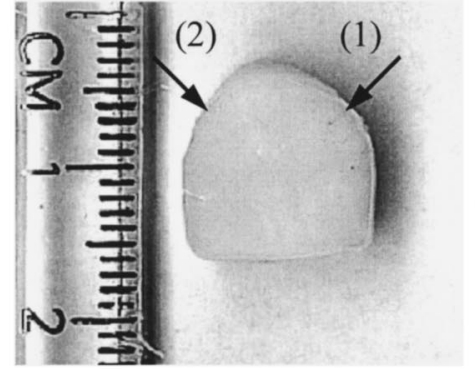

(a)

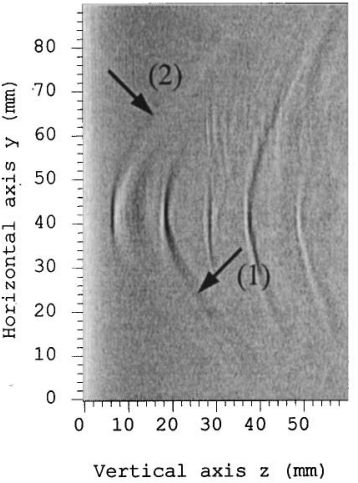

Original signal

(b)

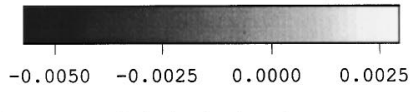

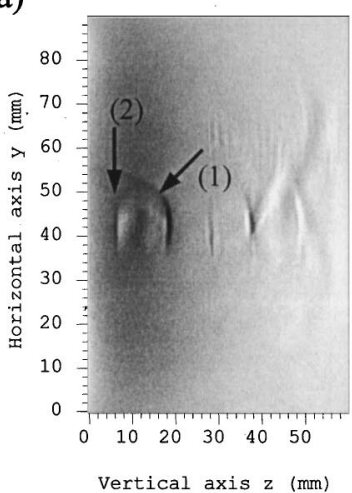

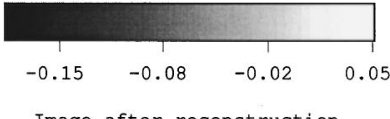

(c)
FIG. 3. (a) Cross-sectional picture of the sample with two curved boundaries marked with (1) and (2); (b) raw data of thermoacoustic signals acquired with the $9 \mathrm{GHz}$ system, where the signals from the two curved boundaries are marked with (1) and (2), respectively; (c) image after the reconstruction, where the images of the two curved boundaries are marked with (1) and (2), respectively.

simple structure to verify the synthetic-aperture method. In the second set of images, we imaged a small fat cylinder containing several detailed structures. The third set of images was acquired, using the $3 \mathrm{GHz}$ system, from two muscle cylinders buried in an ellipse of pork fat. Comparing the last two sets of images demonstrates the effects of different object shapes and the different frequencies of the microwave sources.

\section{A. Results}

The sample in the first set of images is a semicylinder of pork fat immersed in mineral oil. Figure 3(a) shows a cross section of the semicylinder with two curved boundaries marked by (1) and (2). The transducer is on the left side of the picture, pointing to the sample and moving along the ruler. The original signals from the transducer are shown in Fig. 3(b). Because the thermoacoustic waves were propagating almost perpendicularly to the boundaries, the lateral signals (1) and (2) in Fig. 3(b) were from the corresponding curved boundaries (1) and (2) in Fig. 3(a). After reconstruction, the original signals (1) and (2) formed the curved boundaries (1) and (2) of the semicylinder in Fig. 3(c). The synthetic-aperture method was proved to be effective in imaging the curved boundaries. The flat boundary of the semi-

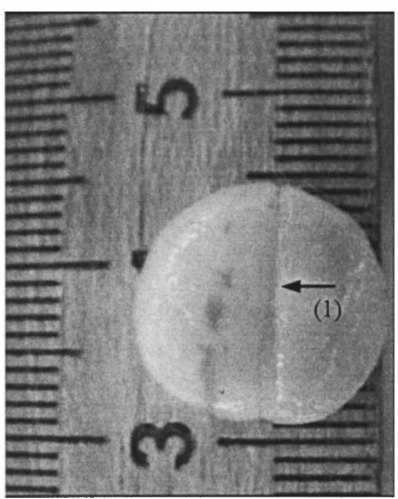

(a)
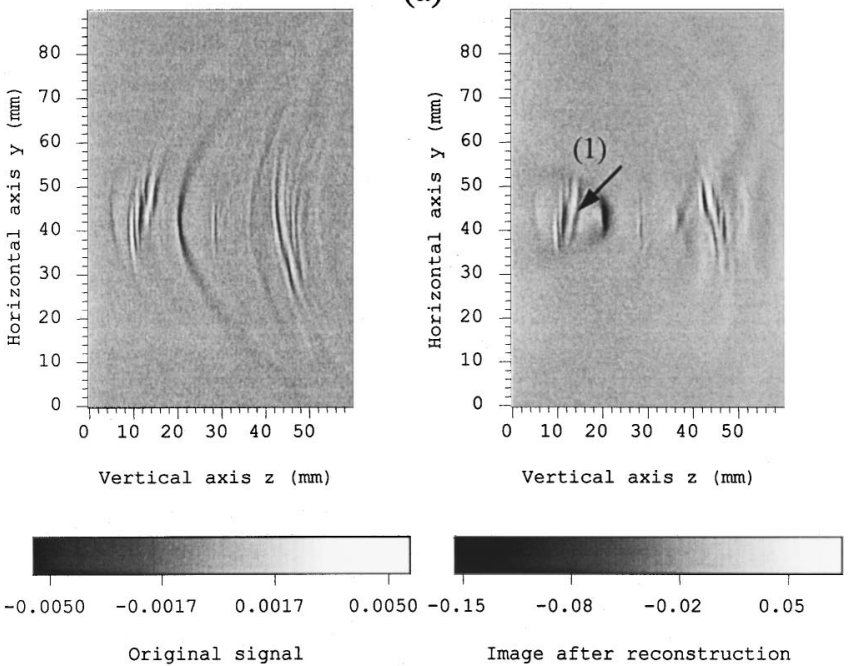

(b)

(c)

FIG. 4. (a) Cross-sectional picture of the sample, a pork fat cylinder with one layer of connective tissue (1) and discrete layers of muscle; (b) raw data of thermoacoustic signals acquired with the $9 \mathrm{GHz}$ system; (c) Image after the reconstruction, where the images of the connective tissue layer is marked with (1).

cylinder is not visible in the image because this boundary was parallel to the axis of the transducer. The thermoacoustic waves from this flat boundary traveled perpendicularly to the axis of the transducer and never reached the transducer.

In the second set of images, Fig. 4(a) is the cross section of the sample, which was a pork fat cylinder with one layer of connective tissue (1) and several small pieces of muscle. The transducer was mounted on the left side of the picture, pointing to the sample and moving along the ruler. The images before and after reconstruction are shown in Fig. 4(b) and Fig. 4(c), respectively. The reconstructed image describes the structure of the sample very well. The connective tissue across the cylinder has been imaged clearly, as marked by (1) in Fig. 4(c). The muscles have been imaged as three slides parallel to each other because of the different distances between the muscles and the transducer. Because of the lateral convolution effect caused by the $6 \mathrm{~mm}$ diam of the transducer, the images of the muscles were stretched and overlapped along the $y$ direction.

In the $9 \mathrm{GHz}$ system, because of the small cross-sectional area in the $9 \mathrm{GHz}$ waveguide, the microwaves were incident 


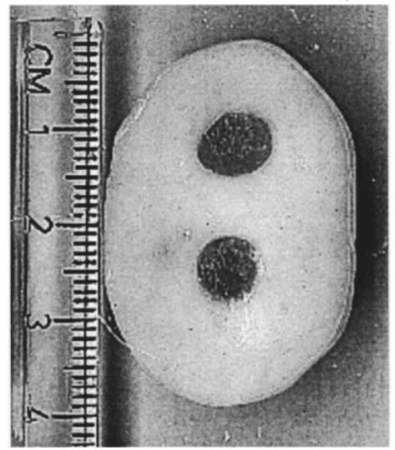

(a)
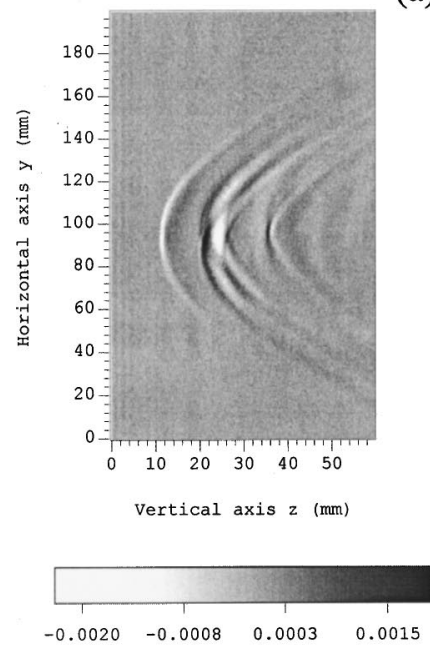

Original Signal

(b)

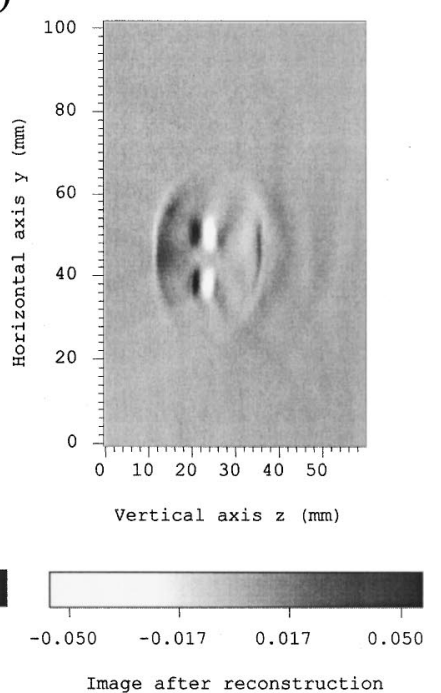

(c)
FIG. 5. (a) Cross-sectional picture of the sample, an ellipse of pork fat containing two muscle cylinders; (b) raw data of thermoacoustic signals acquired with the $3 \mathrm{GHz}$ system; (c) image after the reconstruction.

upon the sample from the right to the left side along the transducer axis but in the opposite direction. In this case, the thermoacoustic waves emitted from the left curved edge were smaller than those emitted from the right curved edge. One reason is that the strong microwave absorption of the sample, especially the muscle layers in the sample in Fig. 4, decreased the intensity of the microwave field on the left side. The other reason is that the distribution of the microwave field, without considering sample absorption, also decreased with increasing distance from the sample to the outlet of the waveguide. Therefore, the left boundary of the sample in Fig. 4(c) is much weaker than the right one.

In the above two images acquired with the $9 \mathrm{GHz}$ system, objects in the right part of the image produce a mirror image due to reflected thermoacoustic waves from the boundaries of the container. The reflected signals could be eliminated by time gating, but for comparison they were kept to maintain the same image scale as the images from the $3 \mathrm{GHz}$ system.

The third set of images shown in Fig. 5 was gathered with the $3 \mathrm{GHz}$ system. Shown in Fig. 5(a) is a cross section of the sample, which was an elliptic slab of pork fat with two muscle cylinders buried inside. With the sample being immersed in the mineral oil, the transducer pointed to the sample from the left side and moved along the ruler. In the 3 $\mathrm{GHz}$ system, the microwaves were incident upon the sample perpendicularly to the picture and pointed outward, which made the microwaves evenly distributed in the cross section of the sample. In the original signal data in Fig. 5(b), the signals from the muscle cylinder and the edge of the pork fat are spread into a hyperbola with similar shapes. The signals from the two muscle cylinders even cross each other in the center. After the reconstruction, the rebuilt image shown in Fig. 5(c) is in good agreement with the real sample shown in Fig. 5(a).

\section{B. Discussion}

From all of the images above, it is clear that the image resolution of curved boundaries of samples and of small cylinders is worse than that of horizontal boundaries, which emit thermoacoustic waves along the transducer axis. For example, the axial resolution of the slabs in Fig. 4(c) is much better than that of the two small cylinders in Fig. 5(c). The reason is that when the thermoacoustic wave does not come from the center of the transducer's receiving solid angle, it may reach different parts of the transducer surface at different times. In this case, the pulse signal is broadened and this broadening of the pulse is proportional to the dimensions of the transducer surface. If the thermoacoustic waves come from the center of the receiving solid angle of the transducer, the resolution is optimized. The propagating direction of most thermoacoustic waves from curved boundaries and small cylinders have large angles with the transducer axis. In other words, when the transducer detects these thermoacoustic waves, they are not from the center of the receiving solid angle. Therefore even after reconstruction, the axial resolution of the curved boundaries or small cylinders has been compromised. Also because of the $6 \mathrm{~mm}$ diam of the transducer, all of the images have been stretched along the $y$ direction and the lateral resolution has deteriorated. Therefore, the diameter of the active element of the transducer is a key to both the axial and lateral resolutions. We can alleviate the effect of stretching and improve the axial resolution of the images by reducing the diameter of the transducer at the cost of losing the SNR.

Comparing the above images before and after reconstruction, the SNR has been greatly improved by the reconstruction. In the delay-and-sum algorithm, the signal intensity of every point is the sum of the signals from different positions that the transducer scanned. From the perspective of the SNR and randomization of the noise, summing up signals from $k$ different positions has the equivalent effect of averaging the signal $k$ times and will increase the SNR by $\sqrt{k}$ times. We can take advantage of this property to greatly reduce our average time in data acquisition and the dose of microwaves.

A comparison of the images in Fig. 4(c) and Fig. 5(c) shows that the $3 \mathrm{GHz}$ system has a much larger image volume than the $9 \mathrm{GHz}$ system due to the deeper penetration depth of the microwaves with a lower frequency and a larger cross section of the waveguide. For microwaves of $3 \mathrm{GHz}$, the penetration depths for muscle and fat are 1.2 and $9 \mathrm{~cm}$, 
respectively, and for microwaves of $9 \mathrm{GHz}$, the penetration depths for muscle and fat are 0.27 and $2.6 \mathrm{~cm}$. The depth we can image in the tissues is proportional to the penetration depth of the microwaves in the tissue. On the other hand, in near-surface imaging the $9 \mathrm{GHz}$ setup has a better SNR than the $3 \mathrm{GHz}$ system due to the larger attenuation of $9 \mathrm{GHz}$ microwaves and the higher-power density of the microwave source.

In traditional LMTT, the ghost caused by the relatively small diameter of the focused transducer affects the lateral resolution. In synthetic aperture, the scanning nonfocused transducer can be artificially focused onto any specified point and the effect equals a focused transducer with a diameter of the scanning dimension. The large diameter, which is much larger than the diameter of the focused transducer used in conventional LMTT, greatly reduces the ghost and, therefore, improves the lateral resolution of the image.

In our study we point out that, compared with traditional LMTT, the synthetic-aperture method is effective for improving the lateral resolution of images and imaging the curved boundaries in samples. The resolution of the images can be further improved by reducing the diameter of the transducer or applying deconvolution with respect to the finite size of the transducer surface.

According to the IEEE standard, our case involves exposures under a controlled environment, which means the exposure is incurred by persons who are aware of the potential of exposure. For both the 3 and $9 \mathrm{GHz}$ microwaves under a controlled environment, the upper limit of safe exposure is $10 \mathrm{~mW} / \mathrm{cm}^{2}$. If it is used for partial body exposure, the upper limit is relaxed to $20 \mathrm{~mW} / \mathrm{cm}^{2}$ for $3 \mathrm{GHz}$ microwaves and $22.1 \mathrm{~mW} / \mathrm{cm}^{2}$ for $9 \mathrm{GHz}$ microwaves. The peak power of our $3 \mathrm{GHz}$ microwave generator is $10 \mathrm{~kW}$; the microwave pulse width is $0.5 \mu \mathrm{s}$; the pulse repetition rate is less than $40 \mathrm{~Hz}$; and the outlet of the microwave generator is $72 \mathrm{~mm}$ $\times 34 \mathrm{~mm}$. As a result, the power density of the $3 \mathrm{GHz}$ microwave system is $8.2 \mathrm{~mW} / \mathrm{cm}^{2}$. The peak power of our 9 $\mathrm{GHz}$ microwave generator is $25 \mathrm{~kW}$; the microwave pulse width is $0.5 \mu \mathrm{s}$; the pulse repetition rate is about $2 \mathrm{~Hz}$; and the outlet of the microwave generator is $23 \mathrm{~mm} \times 10 \mathrm{~mm}$. Consequently, the power density of the $9 \mathrm{GHz}$ microwave system is $10.9 \mathrm{~mW} / \mathrm{cm}^{2}$. Further, we assumed that the entire microwave has been coupled out of the waveguide without divergence. In practice, however, only part of the microwave is coupled out of the waveguide and diverged into a much larger area than the outlet of the waveguide. The power densities used in our experiments are below the limits of the IEEE standard and are safe to humans.

\section{CONCLUSIONS}

The synthetic aperture, which has never been used in LMTT, is proved to be a powerful image reconstruction method. The reconstruction method based on the delay-andsum algorithm has been verified to work well in LMTT because of its ability to image curved boundaries in samples, to improve the lateral resolution, and to reduce the noise of the system. The large diameter of the transducer causes resolu- tion deterioration; the diameter can, however, be reduced to improve the resolution at the expense of the SNR. The comparison of the images shows that the $3 \mathrm{GHz}$ system has a larger imaging volume but a poorer SNR than the $9 \mathrm{GHz}$ system in near-surface imaging.

\section{ACKNOWLEDGMENTS}

This project was sponsored in part by the U.S. Army Medical Research and Material Command Grant No. DAMD17-00-1-0455, the National Institutes of Health Grants No. R01 CA71980 and No. R21 CA83760, the National Science Foundation Grant No. BES-9734491, and Texas Higher Education Coordinating Board Grant No. ARP 000512-0123-1999.

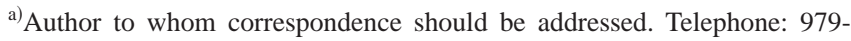
847-9040; fax: 979-845-4450; electronic mail: LWang@tamu.edu; URL: http://oilab.tamu.edu

${ }^{1}$ W. Joines, R. Jirtle, M. Rafal, and D. Schaeffer, "Microwave power absorption differences between normal and malignant tissue," Int. J. Radiat. Oncol., Biol., Phys. 6, 681-687 (1980).

${ }^{2}$ S. Chaudhary, R. Mishra, A. Swarup, and J. Thomas, “Dielectric properties of normal human breast tissues at radiowave and microwave frequencies," Indian J. Biochem. Biophys. 21, 76-79 (1984).

${ }^{3}$ W. Joines, Y. Zhang, C. Li, and R. Jirtle, "The measured electrical properties of normal and malignant human tissues from 50-900 MHz," Med. Phys. 21, 547-550 (1994).

${ }^{4}$ L. E. Larsen and J. H. Jacobi, in Medical Applications of Microwave Imaging (IEEE, Piscataway, NJ, 1986).

${ }^{5}$ S. Caorsi, A. Frattoni, G. L. Gragnani, E. Nortino, and M. Pastorino, "Numerical algorithm for dielectric-permittivity microwave imaging of inhomogeneous biological bodies," Med. Biol. Eng. Comput. 29, NS37-44 (1991).

${ }^{6}$ M. S. Hawley, A. Broquetas, L. Jofre, J. C. Bolomey, and G. Gaboriaud, "Microwave imaging of tissue blood content changes," J. Biomed. Eng. 13, 197-202 (1991).

${ }^{7}$ P. M. Meaney, K. D. Paulsen, and J. T. Chang, "Near-field microwave imaging of biologically-based materials using a monopole transceiver system," IEEE Trans. Microwave Theory Tech. 46, 31-45 (1998).

${ }^{8}$ L.-H. V. Wang, X. Zhao, H. Sun, and G. Ku, "Microwave-induced acoustic imaging of biological tissues," Rev. Sci. Instrum. 70, 3744-3748 (1999).

${ }^{9}$ G. Ku and L.-H. V. Wang, "Scanning thermoacoustic tomography in biological tissue," Med. Phys. 27, 1195-1202 (2000).

${ }^{10} \mathrm{G}$. Ku and L.-H. V. Wang, "Scanning microwave-induced thermoacoustic tomography: signal, resolution, and contrast," Med. Phys. 28, 4-10 (2001).

${ }^{11}$ C. G. A. Hoelen, F. F. M. Demul, R. Pongers, and A. Dekker, "Threedimensional photoacoustic imaging of blood vessels in tissue," Opt. Lett. 23, 648-650 (1998).

${ }^{12}$ C. G. A. Hoelen, R. Pongers, G. Hamhuis, F. F. M. Demul, and J. Greve, "Photoacoustic blood cell detection and imaging of blood vessels in phantom tissue," SPIE 3196, 142-153 (1998).

${ }^{13}$ A. A. Karabutov, E. V. Savateeva, N. B. Podymova, and A. A. Oraevsky, "Backward mode detection of laser-induced wide-band ultrasonic transients with optoacoustic transducer," J. Appl. Phys. 87, 2003-2014 (2000).

${ }^{14}$ R. A. Kruger, P. Liu, Y. R. Fang, and C. R. Appledorn, "Photoacoustic ultrasound (PAUS) — Reconstruction tomography,' Med. Phys. 22, 16051609 (1995). 